\title{
Erratum to: The Political Economy of Britain in Crisis
}

\author{
Christopher Kirkland
}

Erratum to:

C. Kirkland, The Political Economy of Britain in Crisis, Building a Sustainable Political Economy: SPERI Research \& Policy, https://doi.org/10.1007/978-3-319-59238-1

In the original version preface and dedication were missing. These have now been included. 